\title{
Two Dimensional Modeling of Nonuniformly DOPED MESFET UNDER ILLUMINATION
}

\author{
Dr B.K.Mishra ${ }^{1,}$ Lochan Jolly $^{2}$ and Kalawati Patil ${ }^{3}$ \\ ${ }^{1}$ Principal,Thakur College of Engg and Technology, Mumbai, India \\ drbk.mishra@thakureducation.org \\ ${ }^{2,3}$ Department of Electronics and Telecommunication, Thakur College of Engg and \\ Technology, Mumbai, India \\ lochan.jolly@thakureducation.org,kalawati.patil@thakureducation.org
}

\begin{abstract}
A two dimensional numerical model of an optically gated GaAs MESFET with non uniform channel doping has been developed. This is done to characterize the device as a photo detector. First photo induced voltage $\left(V_{o p}\right)$ at the Schottky gate is calculated for estimating the channel profile. Then Poisson's equation for the device is solved numerically under dark and illumination condition. The paper aims at developing the MESFET 2-D model under illumination using Monte Carlo Finite Difference method. The results discuss about the optical potential developed in the device, variation of channel potential under different biasing and illumination and also about electric fields along $X$ and $Y$ directions. The Cgs under different illumination is also calculated. It has been observed from the results that the characteristics of the device are strongly influenced by the incident optical illumination.
\end{abstract}

\section{KEYWORDS}

Optoelectronics, Schottky Junction, Photodetectors, Photovoltage

\section{INTRODUCTION}

Optical control of microwave devices and circuits has been an area of research for the past two decades. In particular, optically controlled GaAs MESFETs (OPFETs) have drawn considerable attention as potential devices in photonic MMICs as well as in OEICs [1].

The key advantage of the MESFET is the higher mobility of the carriers in the channel as compared to the MOSFET [2]. In MESFETs the higher mobility leads to a higher current, transconductance and transit frequency of the device [3,4] making it suitable for high speed MMICs applications. In addition to this, the buried channel in MESFETs yields a better noise performance. This is because trapping and release of carriers, into and from surface states and defects, are eliminated. The use of MESFETs also provides one more significant advantage that it is possible to fabricate semi-insulating (SI) GaAs substrates, which eliminates the problem of absorbing microwave power in the substrate due to free carrier absorption.

As a number of research investigations report that MESFET has the potential for performing optically controlled functions, it can be used to form an additional input port in photonic MMIC [5]. So for the circuit designer there is a necessity of a good model with all major effects considered. This justifies an increased interest in the physical mechanism which occurs in MESFET when illuminated [6].

It is observed that when the gate length becomes shorter than about $2 \mu \mathrm{m}$, two dimensional effects dominates the device operation [7]. Therefore for accuracy while modeling MESFET under illumination both the device structure and the appropriate physical 2-D equations governing device behavior are taken into consideration. 
This paper presents the two-dimensional (2-D) numerical simulation of MESFET using Monte Carlo finite difference method. This technique is used as it helps to simulate correctly complicated 2-D channel potential and electric field equations.

\section{THEORY}

The schematic structure of the MESFET under consideration is shown in the Figure 1.

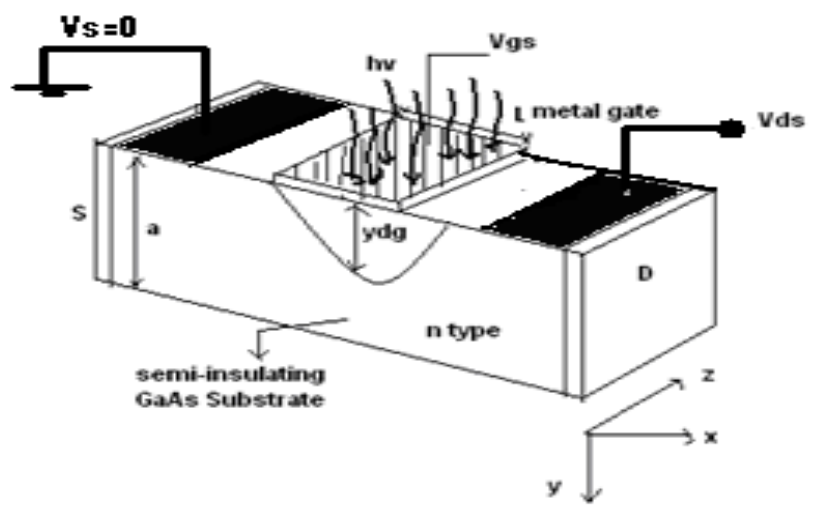

Figure1. Schematic of MESFET under illumination

This is similar to the conventional MESFET except the former uses the semitransparent metal gate which facilitates the absorption of optical radiation illuminated on the gate. For this work we have used nonuniformly doped ion-implanted n-channel MESFET. The optical radiation is incident in the vertical direction y-direction and drain to source current flows in $\mathrm{x}$-direction as shown in Figure. 1.

The two dimensional modeling of MESFET is done using basic two dimensional (2-D) Poisson's equation in the gate-depletion region under the illuminated condition with the Schottky contact $[1,7]$.

$$
\begin{aligned}
& \frac{\partial^{2} \psi}{\partial^{2} X}+\frac{\partial^{2} \psi}{\partial^{2} Y}=\frac{-q}{\varepsilon_{s}}\left[N_{d}(y)+K 1 e^{-\alpha y}\right] \\
& K 1=\frac{P_{o p t}\left(1-R_{m}\right)\left(1-R_{s}\right) \alpha \tau_{L}}{h v}
\end{aligned}
$$

where

$\mathrm{N}_{\mathrm{d}}(\mathrm{y})$ is the non uniform doping density along y direction

$$
N_{d}(y)=\frac{Q}{\sigma \sqrt{2 \pi}} \exp \left(-\left(\frac{y-R p}{\sigma \sqrt{2}}\right)^{2}\right)
$$

where

Q Ion implanted dose

$\sigma \quad$ Straggle parameter

$\mathrm{R}_{\mathrm{p}} \quad$ Projected range.

$\psi \quad$ 2-D potential distribution

$\varepsilon_{\mathrm{s}} \quad$ Permittivity of the GaAs

$R_{m}$ Reflection coefficient at the entrance

$\mathrm{R}_{\mathrm{s}}$ Reflection coefficient at the metal semiconductor contact 
$\mathrm{P}_{\mathrm{opt}}$ Incident optical power density

h Planck's constant

$v$ Frequency of the incident radiation

$\alpha$ Optical absorption coefficient of the semiconductor at the operating wavelength

$\tau_{\mathrm{L}}$ Mean lifetime of the minority carriers under illumination

q Electron charge

\subsection{Calculation of Photovoltage}

Due to illumination, the device performance varies due to the photovoltage developed at the Schottky junction. This photovoltage is calculated using the following relation [1,8] Vop $=\frac{n k T}{q} \ln \left[\frac{q}{J_{S c}} \frac{\alpha P o p t}{h \nu L}\left(\int_{0}^{L} \int_{0}^{y d g(x)} \exp (\alpha y) d y d x\right)\right]$

where

n Ideality factor

k Boltzmann constant

$\mathrm{T}$ Absolute temperature

$\mathrm{Z}$ Width of the gate

L Channel length

$\mathbf{J}_{\mathrm{sc}}$ Reverse saturation current density at Schottky contact

$\mathrm{y}_{\mathrm{dg}}(\mathrm{x})$ Depletion width at $\mathrm{x}$

\subsection{Calculation of Channel Charge}

The total channel charge in the channel is due to the carries present because of ion-implantation and optical generation [2].

$Q_{\text {total }}=Q_{\text {ion }}+Q_{\text {illu }}$

The charge is calculated using the relation [1]

$$
\begin{aligned}
& Q_{n}(V)=q \int_{y_{d g}}^{a} N_{d}(y) d y+q Z(y) \\
& Z(y)=K 1 \int_{0}^{a} \exp (-\alpha y) d y
\end{aligned}
$$

where

$\mathrm{y}_{\mathrm{dg}}$ variation of depletion depth under the gate and is calculated as $[6,8]$.

$y_{d g}(x)=\left[\frac{2 \varepsilon}{q N_{d r}}\left(\phi_{B}-\Delta+V(x)-V_{g s}\right)\right]$

where

$\mathrm{N}_{\mathrm{dr}}$ Constant doping concentration in the active region equivalent ion-implanted

$\mathrm{V}(\mathrm{x})$ Channel voltage at $\mathrm{x}$

$\Phi_{\mathrm{B}} \quad$ Schottky barrier height 
$\Delta \quad$ Position of fermi level at the neutral region below the conduction band

Under illumination $\mathrm{Y}_{\mathrm{dg}}$ is modified to $\mathrm{Y}_{\mathrm{dg}}$ ' given by $[6,8]$

$$
y_{d g}^{\prime}(x)=\left[\frac{2 \varepsilon}{q N_{d r}}\left(\phi_{B}-\Delta+V(x)-V_{g s}-V_{o p}\right)\right]^{1 / 2}
$$

It is the function of potential distribution in the channel.

\subsection{Channel Potential}

The channel voltage profile has been obtained by dividing the channel region into meshes as shown in Figure.3

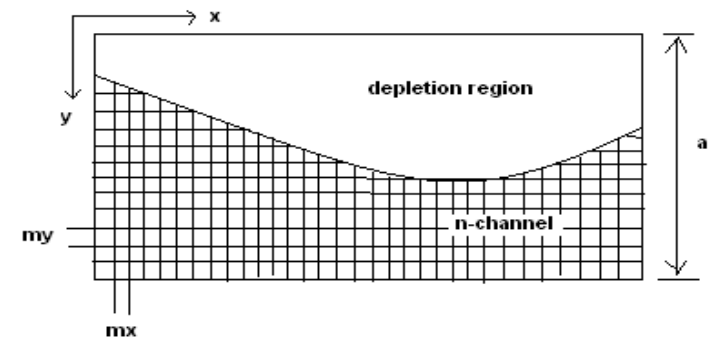

Figure 2. Mesh modeling of the channel region

The potential distributions have been obtained using the appropriate boundary conditions. The potential at subsequent mesh points towards the drain end separated by ' $\mathrm{mx}$ ' in longitudinal direction and 'my' in the transverse direction have been calculated using the discrete form as in equation (10) until the drain end of the gate is reached. To calculate the channel potential Monte Carlo finite difference method is used.

The Monte Carlo finite difference method first forms the grid and then assigns boundary conditions. We are able to calculate the channel potential at every grid point using two iterative methods namely Gaussian iteration process and Liebmann iteration [9].

The solution is computed using the following equation $[1,9,10]$ :

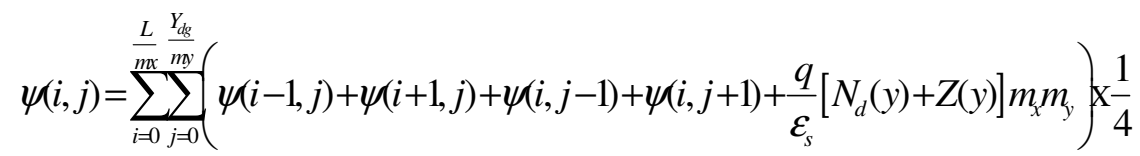

The boundary conditions are taken from [1,11].The effect of illumination is included by replacing Vgs by $\left(\mathrm{V}_{\mathrm{gs}}+\mathrm{V}_{\mathrm{OP}}\right)$,

$$
\begin{aligned}
& \left.\psi(x, y)\right|_{y=0}=V_{g s}+V_{o p}-\phi_{b i} \\
& \left.\psi(x, y)\right|_{x=0}=V_{b i} \\
& \left.\psi(x, y)\right|_{x=L}=V_{b i}+V_{d s} \\
& \left.\psi(x, y)\right|_{y=a}=0
\end{aligned}
$$

where

$\mathrm{V}_{\mathrm{gs}}$ Gate to source voltage

$\mathrm{V}_{\mathrm{ds}}$ Drain to source voltage 
$\Phi_{b i}$ Built in voltage of the Schottky barrier gate

$\mathrm{V}_{\mathrm{bi}}$ Built in voltage between the channels to source junction

\subsection{Electric field calculation}

The electric field along $\mathrm{x}$ and $\mathrm{y}$ direction have been obtained by solving following equations $[9,10]$

$$
\begin{aligned}
& E_{x}=\frac{\psi(i+1, j)-\psi(i-1, j)}{2 m x} \\
& E_{y}=\frac{\psi(i, j+1)-\psi(i, j-1)}{2 m y}
\end{aligned}
$$

These equations have been utilized for estimating the field dependent mobility and the drain current characteristics equation.

\subsection{Mobility model}

The field dependent mobility is given by [12]

$\mu_{n}\left(E_{x}\right)=\mu_{0}+\frac{2\left(-2 \mu_{0} E_{c}+3 v_{\text {sat } 1}\right)}{E_{c}^{2}} E_{x}+\frac{3\left(\mu_{0} E_{c}-2 v_{\text {sat } 1}\right)}{E_{c}^{3}} E_{x}^{2}$

where

$\mu_{\mathrm{o}}$ Low field electron mobility

$\mathrm{V}_{\text {sat } 1}$ Saturation velocity

$\mathrm{E}_{\mathrm{c}}$ Critical field

The velocity-field characteristic of electrons in GaAs is assumed, i.e [13].

$v(E)=\frac{\mu_{0} E+v_{s}\left(E / E_{c}\right)^{4}}{1+\left(E / E_{c}\right)^{4}}$

where

$$
E=\sqrt{E_{x}^{2}+E_{y}^{2}}
$$

\subsection{Gate to source capacitance Cgs model}

The gate to source capacitance can also be calculated using the following relation under dark and illuminated condition $[1,14]$ by calculating the charges under different illuminated condition as given by equation(6).

$$
C_{g s}=\left.\frac{d Q_{d}}{d V_{g s}}\right|_{V_{d s}=c o n s \tan t}=\frac{Q_{d}(i+1)-Q_{d}(i-1)}{V_{g s}(i+1)-V_{g s}(i-1)}
$$

\section{RESULTS AND DISCUSSIONS}

MATLAB simulation has been carried out for GaAs MESFET at $300 \mathrm{~K}$ under dark and illuminated conditions. The gate metallization has been assumed to be thin enough to allow $90 \%$ of the incident radiation to pass through. The parameters used in the calculations are shown in Table 1. 
Table 1. Parameters used in the simulation

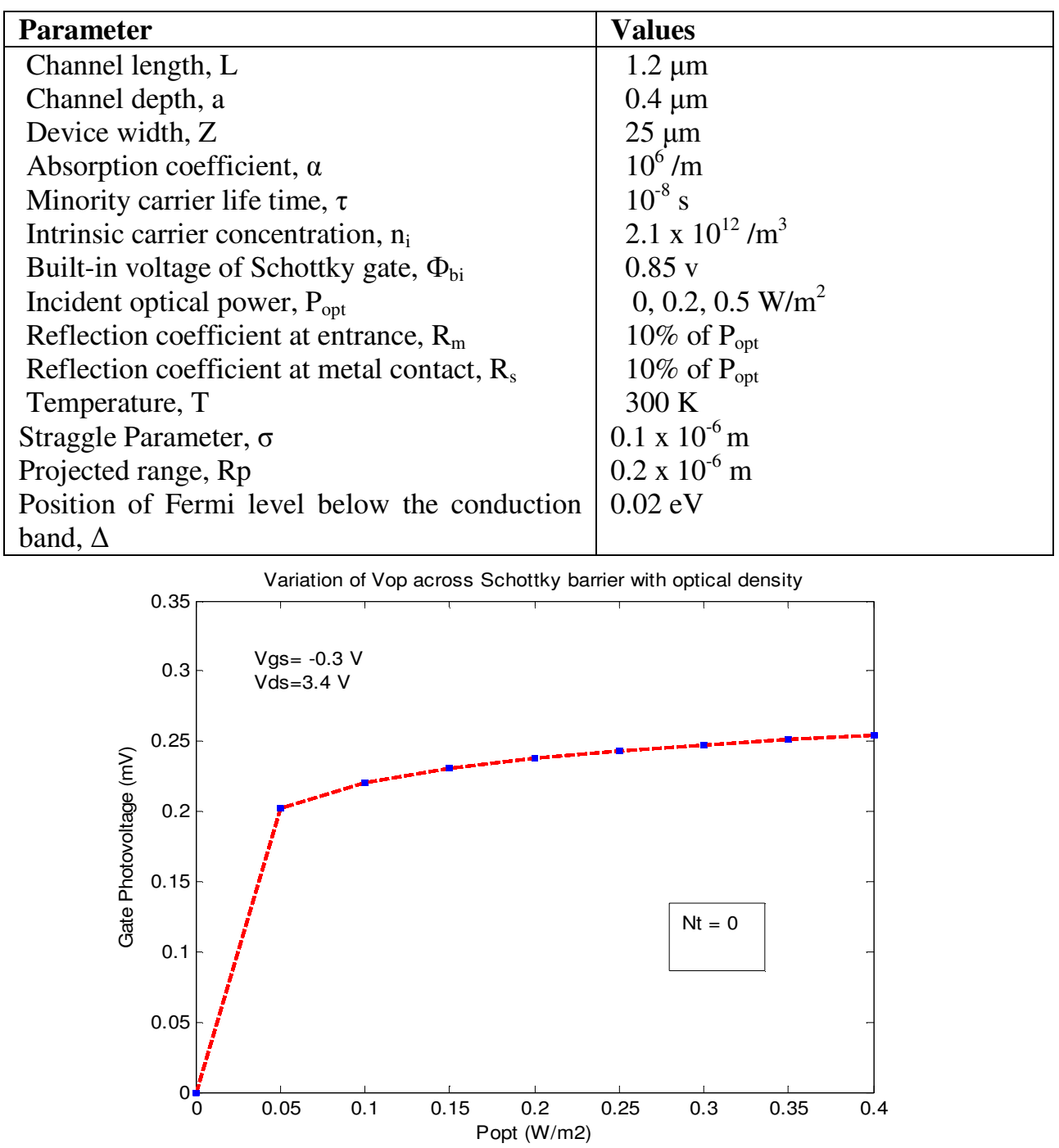

Figure 3. Variation of photovoltage $\mathrm{V}_{\mathrm{op}}$ across the Schottky barrier

Figure 3 shows the variation of the photo voltage developed at the Schottky contact with the optical power density. Surface recombination has not been taken into account. The photo voltage developed at the Schottky junction increases with the incident optical power density Popt, and finally saturates at higher values of optical power density. This saturation at higher values of optical density is due to reduction in the lifetime of the carriers in the presence of illumination, which limits the excess photo generation under the intense illumination. 


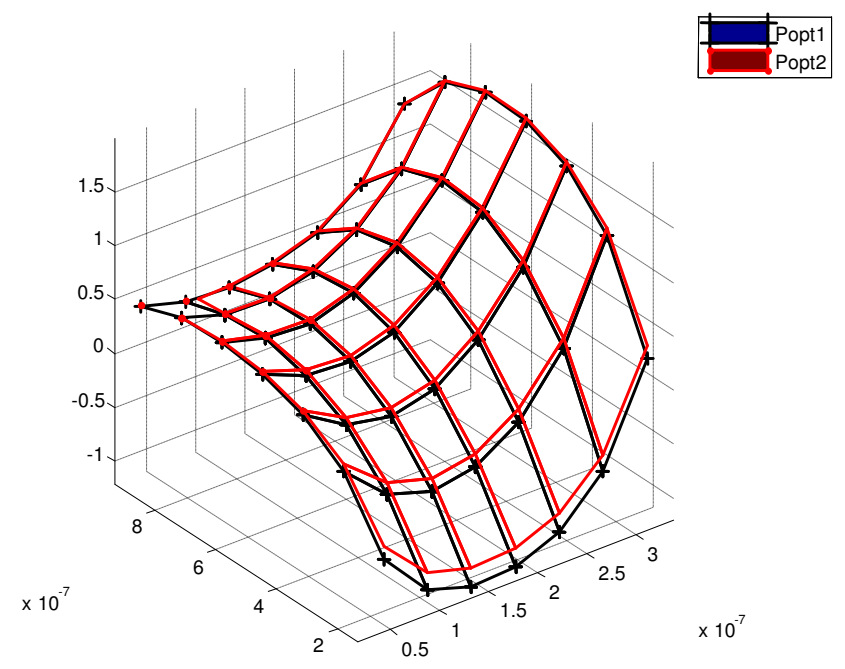

Figure 4. 3-D Plot of channel potential

Figure 4 shows the variation of channel potential with $\mathrm{Lx}$ and $\mathrm{Ly}$ for different illumination $\left(\right.$ Popt $1=0 \mathrm{~W} / \mathrm{m}^{2}$, Popt $\left.2=0.2 \mathrm{~W} / \mathrm{m}^{2}\right)$. It clearly shows that the channel potential increases towards the drain side. This is because the biasing is applied at the drain. These variations can be seen in Figure 7.

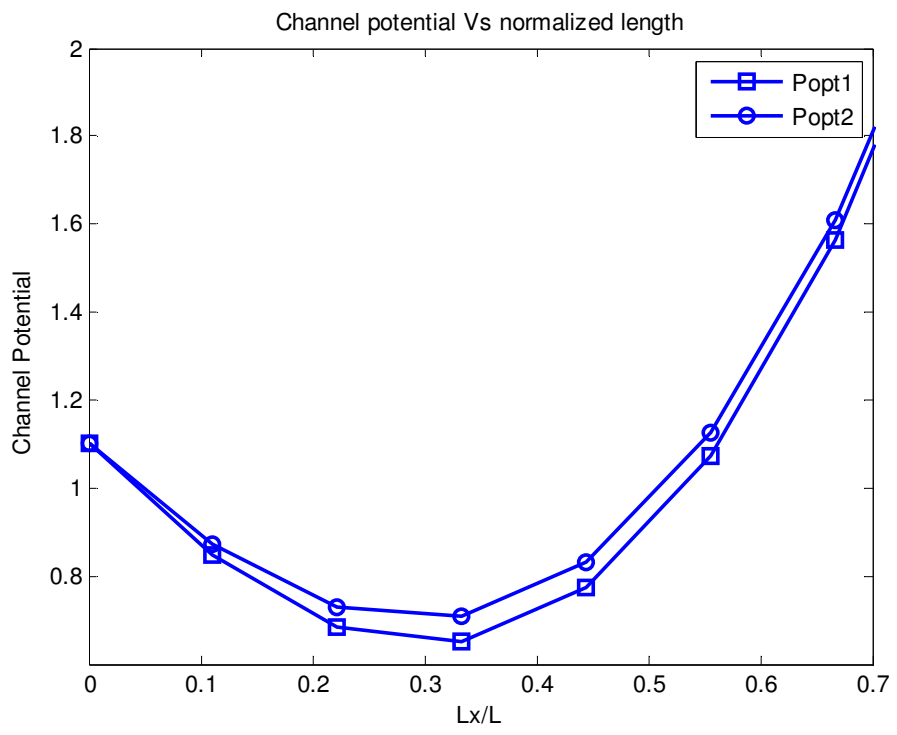

Figure 5. Channel potential Vs normalized distance for various illuminations

Figure 5 shows the variation of the channel potential along the channel length under illumination (Popt $1=0 \mathrm{~W} / \mathrm{m}^{2}$, Popt $2=0.2 \mathrm{~W} / \mathrm{m}^{2}$ ). It can be clearly seen that the channel potential increases with the increase in illumination. This is because with the illumination a forward biasing potential is developed at the gate which increases the channel potential as the illumination increases. 


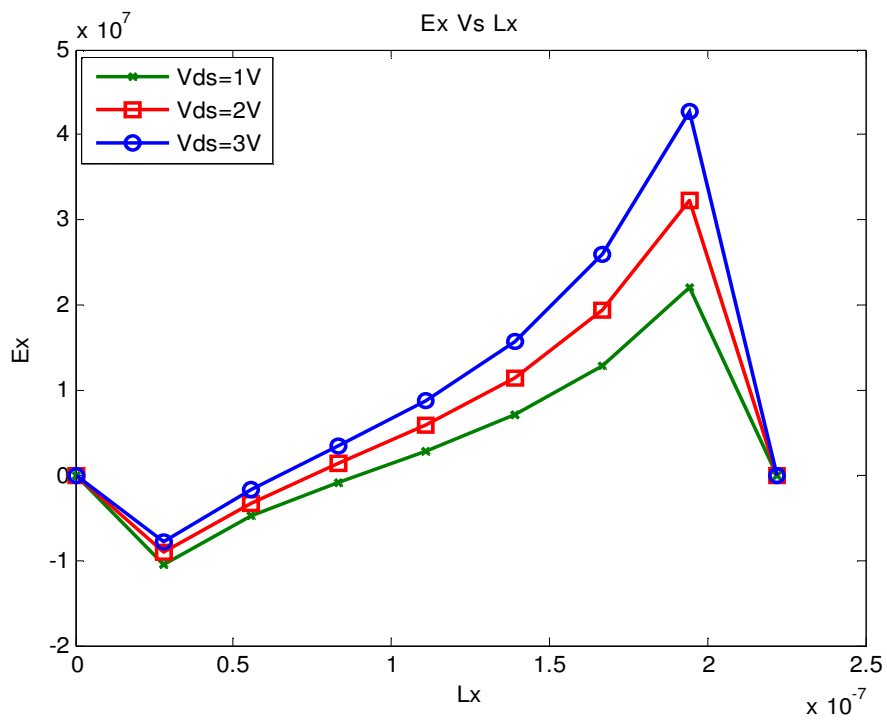

Figure 6. Ex Vs Lx for various Vds

Figure 6 shows the variation of Ex with Lx for various Vds. The plot shows that field is maximum near drain and also the plot shows that Ex increases with Vds and the effect is prominent near the drain. This is because the biasing potential is applied along $\mathrm{X}$ direction and at the drain.

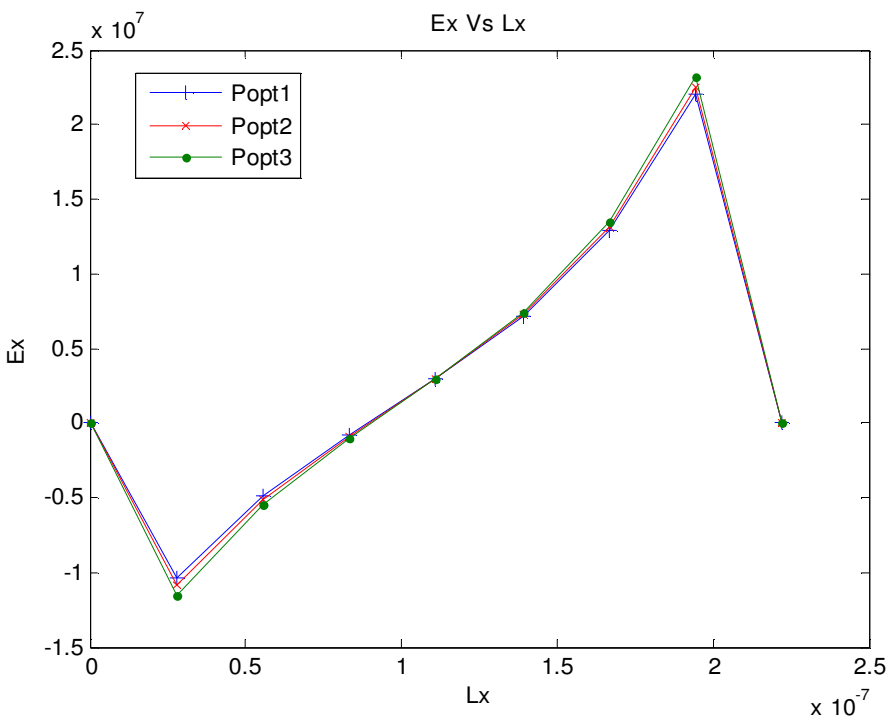

Figure 7. Ex Vs Lx with illumination

Figure 7 shows the variation of Ex with Lx for different illumination (Popt $1=0 \mathrm{~W} / \mathrm{m}^{2}$, Popt $2=0.2 \mathrm{~W} / \mathrm{m}^{2}$, Popt $1=0.5 \mathrm{~W} / \mathrm{m}^{2}$ ). It is observed that there is very small change in the field with illumination. This is because the illumination is applied in the $\mathrm{Y}$ direction. 


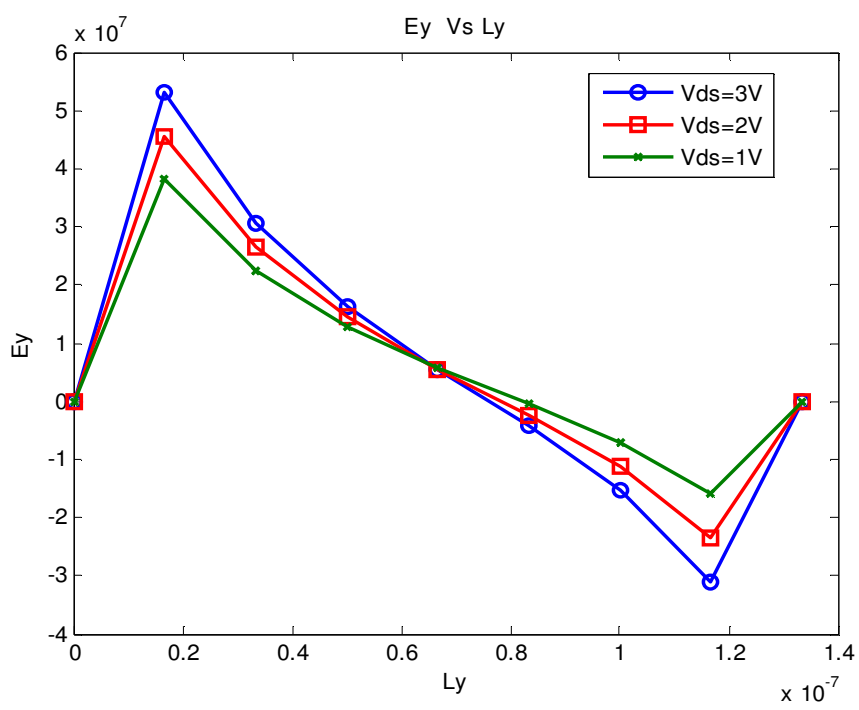

Figure 8. Ey Vs Ly Field for various Vds

Figure 8 shows the variation of Ey with Ly for various Vds. The plot shows that field is maximum in the depletion region and increases with increase in Vds.

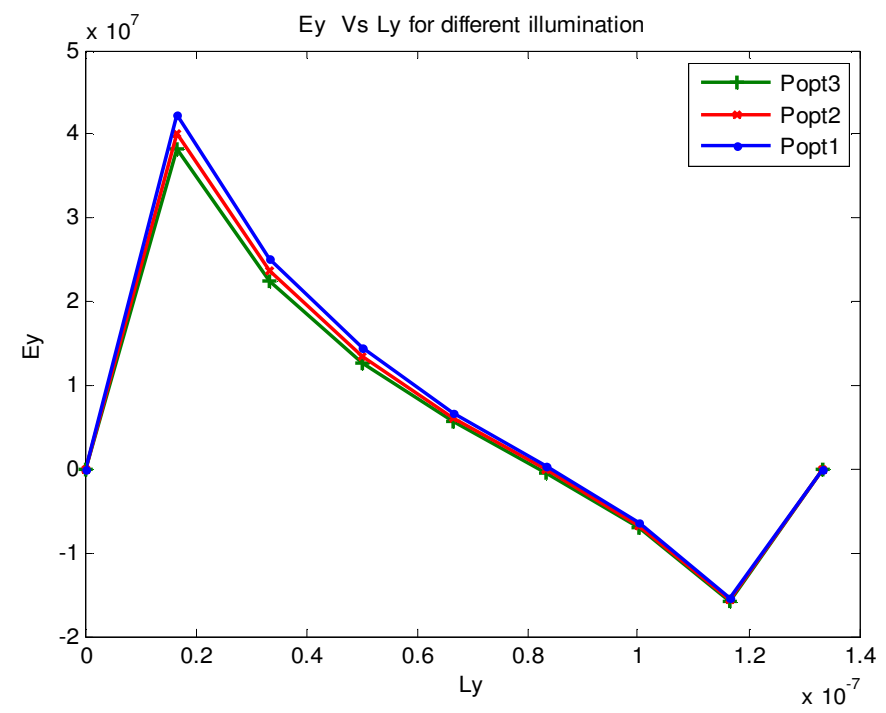

Figure 9. Ey Vs Ly Field for various illumination

Figure 9 shows the variation of Ey with Ly for different illumination (Popt $1=0 \mathrm{~W} / \mathrm{m}^{2}$, Popt $2=0.2 \mathrm{~W} / \mathrm{m}^{2}$, Popt $\left.1=0.5 \mathrm{~W} / \mathrm{m}^{2}\right)$. It is observed that the change in the field with illumination is more prominent as compared with Ex because the field Ey and illumination are along same direction. It is also observed that the effect of illumination is prominent near the source as compared to drain. This is because biasing is applied at the drain. 


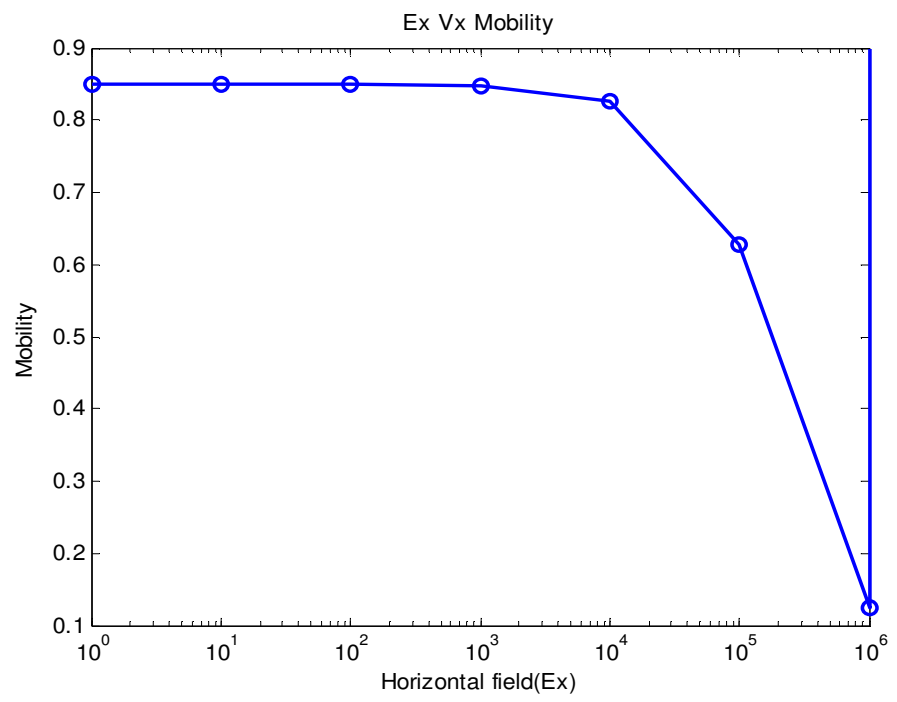

Figure 10. Mobility Vs field

Figure 10 shows the variation in mobility along with the field and shows that the mobility is low field mobility till threshold field and decreases for higher field.

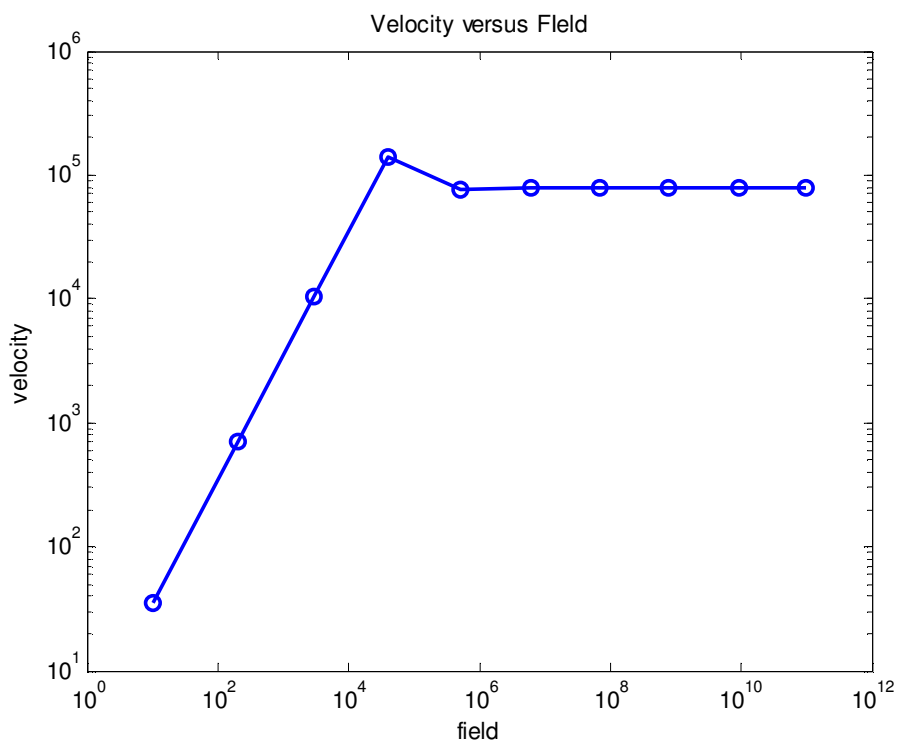

Figure 11. Carrier Velocity Vs Field

Figure 11 shows the variation of the charge velocity with the electric field. It shows that for low field the velocity of the carrier increases with the increase in the field and after threshold field the velocity reaches saturation and remains constant. 


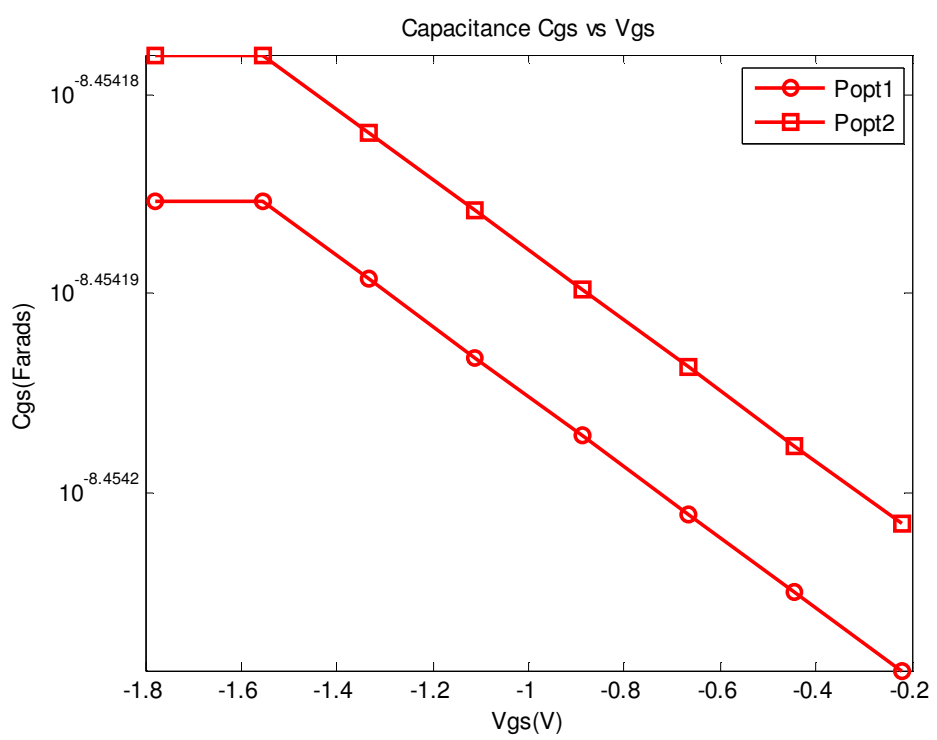

Figure 12. Cgs Vs Vgs

Figure 12 shows the variation of the gate to source capacitance Cgs with the Vgs for different illumination $\left(\mathrm{Popt} 1=0 \mathrm{~W} / \mathrm{m}^{2}\right.$, Popt $\left.2=0.2 \mathrm{~W} / \mathrm{m}^{2}\right)$. It shows that the capacitance increases slightly with the increase in illumination.

\section{VERIFICATION OF RESULTS}

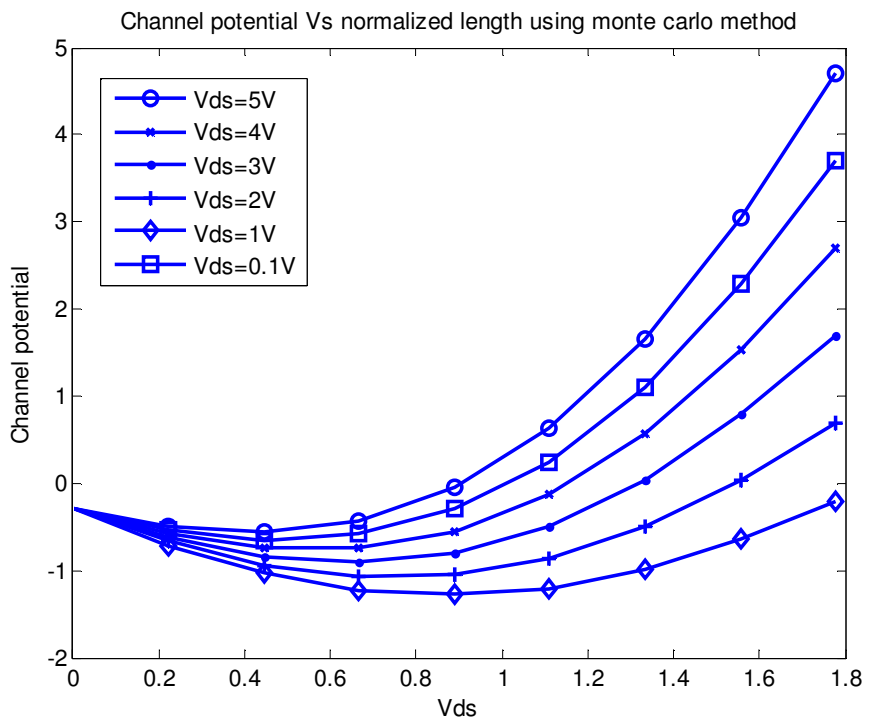

Figure 13. Channel potential Vs normalized length for different illumination

Figure 13 shows the variations of channel potential with normalized length for different Vds .It shows that channel potential increases with increase in Vds. The results presented by this model are same as reported by [11] for same dimensions and biasing condition. 


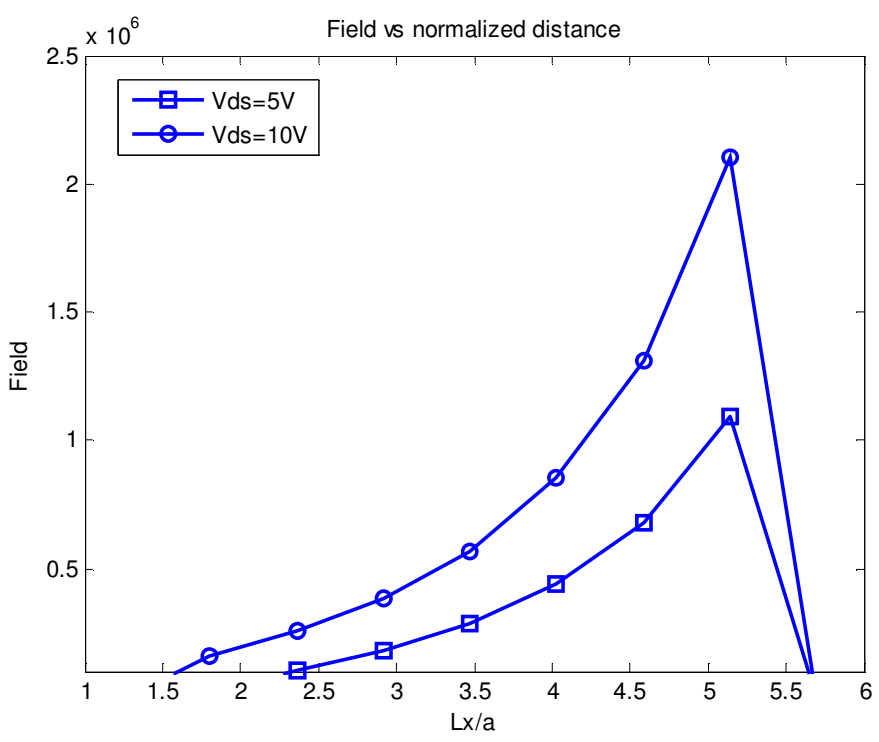

Figure 14. Horizontal field vs normalized length for different Vds

Figure 14 shows Ex with normalized length for different Vds .It shows that field increases with increase in Vds. The results presented by this model are same as reported by [15] for same dimensions and biasing condition.

\section{CONCLUSION}

A 2-D simulation for non-uniformly doped GaAs MESFET with ion-implanted channel profile has been carried out under various illuminated conditions. The model developed solves numerically the basic 2-D semiconductor Poisson's equations. The results show that optical voltage developed in the device increases with the increase in optical power density. The variation of channel potential and electric fields along $\mathrm{X}$ and $\mathrm{Y}$ directions have been simulated under different biasing and illumination conditions .It is observed that both the parameters show positive changes in their values with the increase in illumination. The Cgs under different illumination is also calculated. It also shows an increase in magnitude with increase in illumination. The results obtained from the simulation compares satisfactorily with reported results for similar structures.

\section{REFERENCES}

[1] Madheswaran Muthusamy and Kalaiarasi Kuppusamy, "Numerical simulation of GaAs MESFET photodetector for OEIC receivers", Proc. of SPIE Vol. 5881, 2005.

[2] S. M. Sze ,’Physics of semiconductor devices", 2nd edition., New Delhi, 1982.

[3] Alvaro Augusto A. De Salles, "Optical Control of GaAs MESFETs", IEEE Transactions on Microwave Theory and Techniques, Vol MIT-31, No 10 Oct, 1983.

[4] Youseef Zebda and S. Abu-Helweh, "AC Characteristics of Optically Controlled MESFET (OPFET)", Journal of Lightwave Technilogy, Vol. 15 No 07, July 1997.

[5] Nandita Saha Roy, B.B.Pal and R.U.Khan,, "Frequency dependent characteristics of an ion implanted GaAs MESFET with opaque gate under illumination", Journal of Lightwave Technology, Vol. 18, Issue 2, Feb 2000, pp. 221-229.

[6] B.K.Mishra, "Computer Aided modeling of Solid state photodetectors", Ph.D thesis Birla institute of technology, Mesra,Ranchi ,1995.

[7] M. S. Tyagi, "Introduction to Semiconductor Materials and Devices", New Delhi, 2000.

[8] Nandita Saha Roy, B.B. Pal and R.U. Khan, "Analysis of GaAs OPFET with Improved Optical Absorption under Back Illumination”, Transactions on Electron Devices, Vol No 46, No 12, December 1999.

[9] Dr.B.S.Grewal , "Higher engineering mathematics", Thirty Fifth edition, Khanna Publishers,India,2001. Chung-HSU Chen and David K.Arch, "The Influence of Electric Field and Mobility Profile on GaAs 
MESFET characteristics", IEEE transactions on Electron Devices, Vol. 36, No.11, 1989.

[10]C.Jacoboni and P.Lugli, "Monte Carlo Method for Semi-conductor Device Simulation" , Springer-Verlag Wien-New York,1989.

[11]Shan-Ping Chin and Ching-Yuan Wu, "A New Two-Dimensional Model for the Potential Distribution of Short Gate-Length MESFET's and its Applications”, Journal on Electron Devices,Vol.39, August 1992.

[12]Madheswaran, V. Rajamani and P.Chakrabarti, "Quasi-Two-Dimensional Simulation of an Ion Implanted GaAs MESFET Photodetector, Microwave and optical technology letters", Vol.26, No.4, August 2000.

[13] M.A.R.Al-Mudares, K.W.H.Foulds, "'Physical explanation of GaAs MESFET I/V Characteristics", IEE Proceedings, Vol 30, No.4, August 1983.

[14] P. Chakrabarti, M.Mdheshwaran, A.Gupta, N.A.Khan, "Numerical Simulation of an Ion -Implanted GaAs OPFET", IEEE transactions on Microwave Theory and Techniques, Vol.46, N0.10, 1998.

[15] Chung-HSU Chen and David K.Arch, "The Influence of Electric Field and Mobility Profile on GaAs MESFET characteristics”, IEEE transactions on Electron Devices, Vol. 36, No.11, 1989.

\section{Authors}

B.K.Mishra was awarded PhD degree from Birla institute of technology in 1998.He has 22years of teaching experience. His present research interest focuses on device modeling of optical sensors.

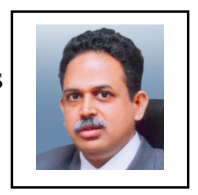

Lochan Jolly completed MTech in 2005 in Microelectronics from IIT Bombay. She has 12 years of teaching experience. She is presently pursuing her Phd. Her present research area is device modeling of MESFET for optical sensor application.

Kalawati Patil is pursuing her post graduation in Electronics \& Telecommunications from Thakur College of Engg. \& Technology, Mumbai. She has 10 years of teaching experience. Her project is on 2-D modeling of GaAs MESFET under optical illumination.
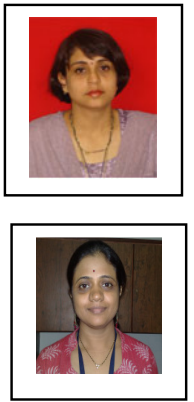